

\title{
Rendering Rough Opaque Materials with Interfaced Lambertian Microfacets
}

Daniel Meneveaux, Benjamin Bringier, Emmanuelle Tauzia, M Ribardière, Lionel Simonot

\section{- To cite this version:}

Daniel Meneveaux, Benjamin Bringier, Emmanuelle Tauzia, M Ribardière, Lionel Simonot. Rendering Rough Opaque Materials with Interfaced Lambertian Microfacets. IEEE Transactions on Visualization and Computer Graphics, 2018, 10.1109/TVCG.2017.2660490 hal-01711532

\section{HAL Id: hal-01711532 \\ https://hal.science/hal-01711532}

Submitted on 18 Feb 2018

HAL is a multi-disciplinary open access archive for the deposit and dissemination of scientific research documents, whether they are published or not. The documents may come from teaching and research institutions in France or abroad, or from public or private research centers.
L'archive ouverte pluridisciplinaire $\mathbf{H A L}$, est destinée au dépôt et à la diffusion de documents scientifiques de niveau recherche, publiés ou non, émanant des établissements d'enseignement et de recherche français ou étrangers, des laboratoires publics ou privés. 


\title{
Rendering Rough Opaque Materials with Interfaced Lambertian Microfacets
}

\author{
Daniel Meneveaux, Benjamin Bringier, Emmanuelle Tauzia, Mickaël Ribardière, Lionel Simonot
}

\begin{abstract}
Specular microfacet distributions have been successfully employed by many authors for representing glossiness of materials. They are generally combined with a Lambertian term to account for the colored aspect. These representations make use of the Fresnel reflectance factor at the interface, but the transmission factor at the interface should also be managed. One solution is to employ a multi-layered model with a single layer for the rough interface, which requires a numerical simulation for handling the multiple reflections of light between the substrate and the interface. In this paper, we propose rather to use a representation corresponding to a Fresnel interface lying on a Lambertian substrate, for which the multiple reflections of light between the interface and the substrate can be expressed analytically. With this interfaced Lambertian model, we show how Fresnel transmission affects the material appearance for flat and rough surfaces with isotropic and anisotropic distributions, that produce light backscattering effects. We also propose a methodology for using such materials in any physically based Monte Carlo rendering system, as well as an approximate representation, suitable for GPU applications or measured data fitting. Our approach generalizes several previous models, including flat Lambertian materials as well as specular and Lambertian microfacets. Our results illustrate the wide range of materials that can be rendered with this representation.
\end{abstract}

Index Terms—surface appearance, BRDF, microfacets, importance sampling
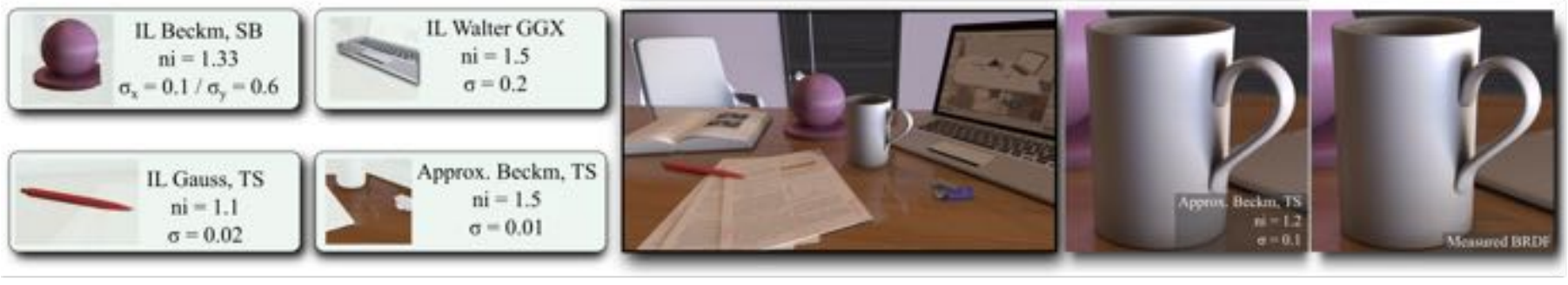

Figure 1: Rough interfaced Lambertian materials with various parameter values, and comparison with fitted measured materials for a ceramic mug. Our model generalizes and subsumes several previous existing models: Lambert, Cook-Torrance [1], and Oren-Nayar [2].

\section{INTRODUCTION}

Realistic and physically plausible representations of reflectance have been studied for a long time in optics and computer graphics. Yet, many questions still remain open because the mathematical models dedicated to Bidirectional Reflectance Distribution Functions (BRDFs) must fulfill various requirements, such as physical plausibility, ability to naturally represent existing/measured materials, visual realism, computation efficiency for producing computer generated images, material control for artists, etc.

- Daniel Meneveaux: XLIM Institute UMR CNRS 7252, University of Poitiers. E-mail: daniel.meneveaux@univ-poitiers.fr

- Benjamin Bringier: XLIM Institute UMR CNRS 7252, University of Poitiers.E-mail: benjamin.bringier@univ-poitiers.fr

- Emmanuelle Tauzia: XLIM Institute UMR CNRS 7252, University of Poitiers. E-mail: emmanuelle.tauzia@univ-poitiers.fr

- Mickaël Ribardière: XLIM Institute UMR CNRS 7252, University of Poitiers. E-mail: mickael.ribardiere@univ-poitiers.fr

- Lionel Simonot: PPRIME Institute UPR CNRS 3346, University of Poitiers. E-mail: lionel.simonot@univ-poitiers.fr
The wide variety of empirical models, introduced for instance by Phong [3], Blinn-Phong [4], or the revised version proposed by Lewis [5], offers a competitive and intuitive way to define surface optical properties, but their parameters do not correspond to actual physical characteristics and they have limited expressiveness. Other approaches favor function bases whose coefficients can be obtained from measurements using projection or parameter fitting, e.g., with wavelets [6], spherical harmonics [7], or Phong lobes [8]. Unfortunately with such representations, both the nature and large number of coefficients make it difficult for users to manually tune material appearances. Finally, physically based BRDF models [1], [2], [9]-[14] are often preferred because they are defined by parameters that correspond to physical aspects of materials, such as surface roughness, chromaticity, or refractive indices.

Among these latter models, distributions of microfacets are often used because they represent a wide range of materials. They have been introduced in computer graphics to capture glossy reflections on rough opaque 
surfaces [1], [15]. These materials can be metallic (complex refractive index), highly reflecting light [16], [17], or dielectric (real refractive index) that only partly reflect light. In this latter case, transmission should be accounted for. For instance, Walter et al. [11] propose to handle refraction of rough transparent dielectrics. With materials such as paints, plastics, ceramics, and so on, light goes through the dielectric interface and scatters within the material body before re-exiting the surface. This effect is often approximated using a Lambertian term independent of the specular aspect of the surface. Two important remarks can be made.

1) A constant Lambertian term combined with specular microfacets does not ensure energy conservation because the specular albedo increases with the incidence direction. Some authors have proposed to overcome empirically this problem [18], [19]. Although physically plausible, this latter representation does not explicitly correlate reflectance with the substrate roughness.

2) The matte component of rough materials is not constant and often exhibits backscattering at grazing incident directions, as first described by Oren and Nayar [2] and also modeled by others [20], [21]. This phenomenon can be observed for instance on the moon appearance, which looks flatter than a Lambertian sphere. Several surfaces exhibiting backscattering are presented in this paper and in the supplemental material.

Several authors have proposed to capture light scattering with multi-layered representations [12], [22], [23], while handling multiple light reflections with rough interfaces, but the translucent medium underneath is considered as flat and therefore uncorrelated from the interface roughness, which also prevents backscattering effects. In addition, these models require precomputations for handling light multiple scattering within layers.

Simonot [24] tackles this problem by introducing interfaced Lambertian microfacets for physically handling transmission through a single Fresnel interface and multiple reflections with a Lambertian substrate. In this case, the rough Fresnel interface fits perfectly the Lambertian substrate, contrary to multi-layered representations for which the successive interfaces are considered as layers and managed as uncorrelated. This theoretical model has been originally defined with a Gaussian distribution of normals, without taking masking and shadowing into account. In addition, similarly to Oren and Nayar's representation [2] all the microfacets contribute to the $\mathrm{BRDF}$, and their use in a rendering framework is not straightforward due to numerical integration.

In this paper, we extend Simonot's work [24] and propose a general methodology for rendering any type of opaque microfacet distributions. We render the appearance of interfaced Lambertian rough surfaces, and compare our results with other BRDF models. Figure 1 illustrates some results produced with the approaches described in this paper. With our model, explicit lighting simulation within layers is not required since light multiple reflections between the substrate and the interface are analytically described. Our methodology directly integrates this representation in any Monte Carlo based lighting simulation renderer. We also propose an approximate model that handles anisotropic Gaussian or Beckmann distributions.

More specifically, the contributions of this paper consist of:

- A consistent framework dedicated to microfacet BRDFs, that integrates a homogeneous matte opaque body with a rough interface. It generalizes several existing models, including flat specular or Lambertian materials, as well as a range from specular to Lambertian rough surfaces, with various distributions, and the corresponding geometric attenuation factors.

- A practical solution for straightforwardly integrating such models within Monte Carlo based rendering systems, including importance sampling, anisotropy, and light scattering within microfacets.

- An approximate model which can be employed for direct lighting, GPU, and measurement fitting. It is designed for several distributions and handles anisotropy.

Our results show that this model accounts for many reflection phenomena, including backscattering, anisotropy, and BRDF darkening at incoming and outgoing grazing angles, that can be observed on many measured materials. These effects are naturally included in the model since it physically handles the balance between specular and body reflections.

The remainder of the paper is organized as follows. Section 2 presents the theory of microfacet BRDF models and introduces the notation. Section 3 describes interfaced Lambertian microfacet BRDFs. Section 4 explains how scattering microfacets can be employed with various configurations in rendering systems. Section 5 presents our results for various cases, including comparisons and fittings from measured BRDFs. Conclusion and future work are provided in Section 6.

\section{Microfacet-Based BRDF Models}

This paper focuses on microfacet-based models for opaque materials, as described by Torrance and colleagues [1], [15], Oren and Nayar [2], Ashikhmin et al. [10], Bagher et al. [25], Dupuy et al. [26], or Heitz [27]. They have been successful at representing various families of materials with only a few parameters clearly related to surface observations and intuitive to manipulate. So far, they have been described using (i) a microfacet distribution, (ii) an elementary reflectance (purely specular or 
Lambertian), and (iii) geometric factors accounting for light shadowing and masking.

\subsection{Definitions and Notation}

The notation used in this paper is given in Table 1 , it corresponds to the one proposed by Nicodemus et al. [28]. Any direction $\mathbf{u}$ is defined with a polar angle $\theta_{u}$ and an azimutal angle $\varphi_{u}$ expressed according to the macrosurface normal $\mathbf{n}$.

Let us consider a surface sample of normal $\mathbf{n}$ lit by a collimated light source from direction $\mathbf{i}$, as illustrated in Figure 2. The radiance reflected toward an observer direction $\mathbf{o}$ is given by the $\operatorname{BRDF} f(\mathbf{i}, \mathbf{o}, \mathbf{n})$. It is defined as the ratio between the elementary radiance $d L(\mathbf{i}, \mathbf{o}, \mathbf{n})$ reflected by the surface in direction $\mathbf{o}$ and the incident irradiance $d E(\mathbf{i}, \mathbf{n})$ coming from direction $\mathbf{i}$ :

$$
f(\mathbf{i}, \mathbf{o}, \mathbf{n})=\frac{d L(\mathbf{i}, \mathbf{o}, \mathbf{n})}{d E(\mathbf{i}, \mathbf{n})} .
$$

A BRDF should be physically plausible, i.e., it should satisfy Helmoltz reciprocity, i.e., $f(\mathbf{i}, \mathbf{o}, \mathbf{n})=f(\mathbf{o}, \mathbf{i}, \mathbf{n})$, and energy conservation, i.e.,

$$
\int_{\Omega_{+}} f(\mathbf{i}, \mathbf{o}, \mathbf{n})|\mathbf{o n}| d \omega_{o} \leq 1, \quad \forall \mathbf{i} \in \Omega_{+} .
$$

The microfacet representation of a BRDF $f(\mathbf{i}, \mathbf{o}, \mathbf{n})$ corresponds to a statistical description of a microfacet distribution. Given the BRDF $f^{\mu}(\mathbf{i}, \mathbf{o}, \mathbf{m})$ of an individual microfacet associated with a normal $\mathbf{m}$, its contribution is weighted by the distribution $D(\mathbf{m})$ and a geometric attenuation factor (or GAF) $G(\mathbf{i}, \mathbf{o}, \mathbf{m})$. The distribution function $D(\mathbf{m})$ defines the surface roughness, indicating the proportion of microfacets oriented according to a given orientation $\mathbf{m}$; the attenuation factor $G(\mathbf{i}, \mathbf{o}, \mathbf{m})$ determines the portion of a microfacet of normal $\mathbf{m}$ visible from both the light source and the observer; it has a major influence at grazing angles. Many authors have studied the use of various distributions and geometric attenuation factors [1], [2], [10], [11], [15], [19], [25], [26], [29], which have to be carefully chosen together [10], [27]. The equation for the general case of microfacet based BRDFs is:

$$
f(\mathbf{i}, \mathbf{o}, \mathbf{n})=\int_{\Omega_{+}} \frac{|\mathbf{i m}|}{|\mathbf{i n}|} f^{\mu}(\mathbf{i}, \mathbf{o}, \mathbf{m}) \frac{|\mathbf{o m}|}{|\mathbf{o n}|} D(\mathbf{m}) G(\mathbf{i}, \mathbf{o}, \mathbf{m}) d \omega_{m} .
$$

All microfacets are supposed to be oriented in the upper hemisphere, so $D(\mathbf{m})=0$ if $\mathbf{m} \cdot \mathbf{n} \leq 0$. The projected areas of all microfacets have to be equal to the macroscopic surface, i.e., $\int_{\Omega_{+}} D(\mathbf{m})|\mathbf{m n}| d \omega_{m}=1$.

In the case of purely specular microfacets, Equation 3 simplifies to [1], [11], [15]:

$$
f(\mathbf{i}, \mathbf{o}, \mathbf{n})=\frac{F(\mathbf{i}, \mathbf{h}) D(\mathbf{h}) G(\mathbf{i}, \mathbf{o}, \mathbf{h})}{4|\mathbf{i n}||\mathbf{o n}|},
$$

where $\mathbf{h}=\frac{\mathbf{i}+\mathbf{o}}{\|\mathbf{i}+\mathbf{o}\|}$ is the half angle vector between $\mathbf{i}$ and $\mathbf{o}$, and $F(\mathbf{i}, \mathbf{h})$ corresponds to Fresnel's reflectance, depending on the refractive index $n_{i}$ (see Table 1 ). This equation defines the glossy aspect of the surface.



Fig. 2: Geometry of reflection with our notation.

TABLE 1: Notation used in this paper.

\begin{tabular}{|c|l|}
\hline $\mathbf{n}$ & Macroscopic normal unit vector \\
$\mathbf{m}$ & Microfacet normal unit vector \\
$\mathbf{i}$ & Incident light unit vector direction \\
$\mathbf{o}$ & Observer unit vector direction \\
$\mathbf{h}$ & Half angle unit vector, bisector between $\mathbf{i}$ and $\mathbf{o}$ \\
$|\mathbf{u v}|$ & $|\mathbf{u v}|=\mathbf{u} \cdot \mathbf{v}$ if $\mathbf{u} \cdot \mathbf{v}>0$ and 0 otherwise \\
$\theta_{u}$ & Polar angle associated with direction $\mathbf{u}$ \\
$\varphi_{u}$ & Azimuthal angle associated with direction $\mathbf{u}$ \\
$d \omega_{u}$ & Elementary solid angle around direction $\mathbf{u}$ \\
$\Omega_{+}$ & Upper hemisphere of direction $\mathbf{n}$ \\
$f(\mathbf{i}, \mathbf{o}, \mathbf{n})$ & Macroscopic BRDF \\
$f^{\mu}(\mathbf{i}, \mathbf{o}, \mathbf{m})$ & Microfacet BRDF \\
$D(\mathbf{m})$ & Microfacet normal distribution \\
$\sigma_{x}, \sigma_{y}$ & Roughness parameters given in local coordinate \\
$G(\mathbf{i}, \mathbf{o}, \mathbf{m})$ & system for dealing with anisotropy \\
$n_{0}$ & Geometric attenuation factor \\
$n_{1}$ & External refractive index \\
$n_{i}=\frac{n_{1}}{n_{0}}$ & Internal refractive index \\
$F(\mathbf{i}, \mathbf{m})$ & Ratio of refractive indices \\
$T(\mathbf{i}, \mathbf{m})$ & Fresnel term, interface reflectance \\
\hline
\end{tabular}

\subsection{Discussion}

On the one hand, metallic surfaces highly reflect light (Fresnel reflectance values are close to 1 , with a wavelength dependency that cannot be neglected), and such rough surfaces can be modeled using only specular microfacets. On the other hand, with dielectrics the specular reflection is nearly constant according to wavelength, and for common values of $n_{i}$ (i.e., $1 \leq n_{i}<3$ ), the Fresnel reflectance is low except at grazing angles.

Considering energy conservation, the interface transmittance is therefore significant although it is generally ignored [1] or empirically approximated [19]. The model presented in this paper explicitly deals with transmission of light through the interface represented by a real refractive index and multiple light reflections between a Lambertian substrate and the interface. 
Oren and Nayar [2] have introduced Lambertian microfacet BRDFs. The objects represented by their model cannot exhibit any glossy appearance, while the CookTorrance model [1] only employs a constant Lambertian term, that does not account for backscattering with rough materials [2]. The strong assumption of considering only pure Lambertian or pure specular microfacets restricts the range of materials that can be reproduced. These models should not be combined for taking into account simultaneously glossy and matte aspects of reflectance for three main reasons: first, energy conservation is not handled with such a combination since body reflection should also depend on $n_{i}$; second, interfaced Lambertian microfacets exhibit chromaticity variations according to the interface refractive index discontinuity, that cannot be handled using a simple combination of models; and third, Oren and Nayar use a distribution that is very rarely used in other models.

This paper provides an answer to the aforementioned issues, and proposes several practical solutions for the implementation which go beyond this specific model since they can be used with any microfacet based BRDFs.

\section{INTERFACED LAMBERTIAN BRDF}

We consider rough surfaces consisting of a Lambertian substrate of parameter $K_{d}$ (dependent on wavelength $\lambda$ ), covered with a flat interface corresponding to a refractive index discontinuity $n_{i}$ (a real index for dielectrics) between these two media (Figure 3). This model accounts for both surface and body reflections, and it should not be considered as a multi-layered representation.

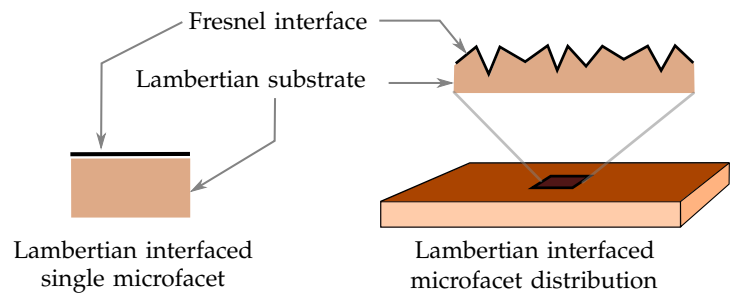

Fig. 3: Surface built up with interfaced Lambertian microfacets. The substrate scatters light while the interface provides brightness.

\subsection{Single Microfacet (or Flat Surface)}

Microfacets are associated with a BRDF $f^{\mu}(\mathbf{i}, \mathbf{o}, \mathbf{m})$ defined by a pure specular interface reflection $f_{s}^{\mu}(\mathbf{i}, \mathbf{o}, \mathbf{m})$ combined with the material body diffuse reflection $f_{b}^{\mu}(\mathbf{i}, \mathbf{o}, \mathbf{m})$ that accounts for light transmission through the interface, light scattering underneath, and light transmission again outside the material:

$$
f^{\mu}(\mathbf{i}, \mathbf{o}, \mathbf{m})=f_{s}^{\mu}(\mathbf{i}, \mathbf{o}, \mathbf{m})+f_{b}^{\mu}(\mathbf{i}, \mathbf{o}, \mathbf{m}) .
$$

Since each microfacet interface is considered flat, the surface reflection component $f_{s}^{\mu}(\mathbf{i}, \mathbf{o}, \mathbf{m})$ is [11]:

$$
f_{s}^{\mu}(\mathbf{i}, \mathbf{o}, \mathbf{m})=F(\mathbf{i}, \mathbf{h}) \frac{\delta \omega_{m}(\mathbf{h}, \mathbf{m})}{4|\mathbf{i h}|^{2}},
$$

where $\mathbf{h}$ is the bisector direction of $\mathbf{i}$ and $\mathbf{o}, \delta \omega_{m}$ is the dirac distribution associated with the elementary solid angle $d \omega_{m}$ around $\mathbf{m}$, so that for any solid angle $\Omega$ :

$$
\int_{\Omega} \delta \omega_{m}(\mathbf{s}, \mathbf{m}) d \omega_{m}= \begin{cases}1 & \text { if } \mathbf{s} \in \Omega \\ 0 & \text { otherwise }\end{cases}
$$

and $F(\mathbf{i}, \mathbf{h})$ is the reflectance of the interface (for unpolarized irradiance) between a medium of index $n_{0}$ and a medium of index $n_{1}\left(n_{i}=n_{1} / n_{0}\right)$ :

$$
F(\mathbf{i}, \mathbf{h})=\frac{1}{2} \frac{(g-c)^{2}}{(g+c)^{2}}\left\{1+\frac{[c(g+c)-1]^{2}}{[c(g-c)+1]^{2}}\right\},
$$

where $c=\mathbf{i} \cdot \mathbf{h}$ and $g^{2}=n_{i}^{2}+c^{2}-1$.

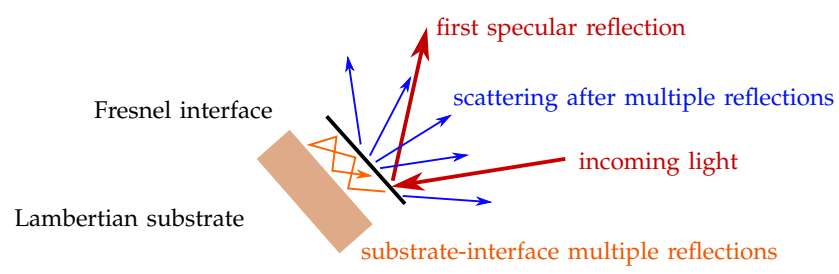

Fig. 4: Light reflection for a single microfacet: light is both reflected and transmitted by the interface, and light scatters multiple times between the body and the inner side of the interface.

As detailed by Elias et al. [30], body scattering (Figure 4) should account for the first interface transmission $T(\mathbf{i}, \mathbf{m})=1-F(\mathbf{i}, \mathbf{m})$ of light, followed by a Lambertian reflection due to the substrate (of reflectance $K_{d}$ ), inner multiple interactions between interface and substrate $1 /\left(1-K_{d} r_{i}\right)$, and final transmissions toward the outgoing direction, of transmittance $T(\mathbf{o}, \mathbf{m})$ :

$$
f_{b}^{\mu}(\mathbf{i}, \mathbf{o}, \mathbf{m})=\frac{1}{\pi n_{i}^{2}} T(\mathbf{i}, \mathbf{m}) T(\mathbf{o}, \mathbf{m}) \frac{K_{d}}{\left(1-K_{d} r_{i}\right)},
$$

where $r_{i}$ is the internal reflectance on the flat interface lit by a Lambertian source coming from the medium of index $n_{1}$ :

$$
r_{i}=\int_{\theta_{u}=0}^{\frac{\pi}{2}} F_{i}(\mathbf{u}, \mathbf{m}) \sin \left(2 \theta_{u}\right) d \theta_{u},
$$

with $F_{i}$ the microfacet inner Fresnel reflectance. In practice, this latter integral can be expressed analytically using the relation $n_{i}^{2}\left(1-r_{i}\right)=1-r_{e}$, where $r_{e}$ is the external reflectance on the flat interface lit by a Lambertian source coming from the medium of index $n_{0}$; Molenaar et al. [31] provide the following expression for $r_{e}$ :

$$
\begin{aligned}
r_{e}= & \frac{1}{2}-\frac{2 n_{i}^{3}\left(n_{i}^{2}+2 n_{i}-1\right)}{\left(n_{i}^{2}+1\right)\left(n_{i}^{4}-1\right)}+\frac{\left(n_{i}-1\right)\left(3 n_{i}+1\right)}{6\left(n_{i}+1\right)^{2}}+ \\
& \frac{8 n_{i}^{4}\left(n_{i}^{4}+1\right)}{\left(n_{i}^{2}+1\right)\left(n_{i}^{4}-1\right)^{2}} \ln \left(n_{i}\right)+\frac{n_{i}^{2}\left(n_{i}^{2}-1\right)^{2}}{\left(n_{i}^{2}+1\right)^{3}} \ln \left(\frac{n_{i}-1}{n_{i}+1}\right) .
\end{aligned}
$$

With interfaced Lambertian microfacets, $1 / n_{i}^{2}$ (Equa- 


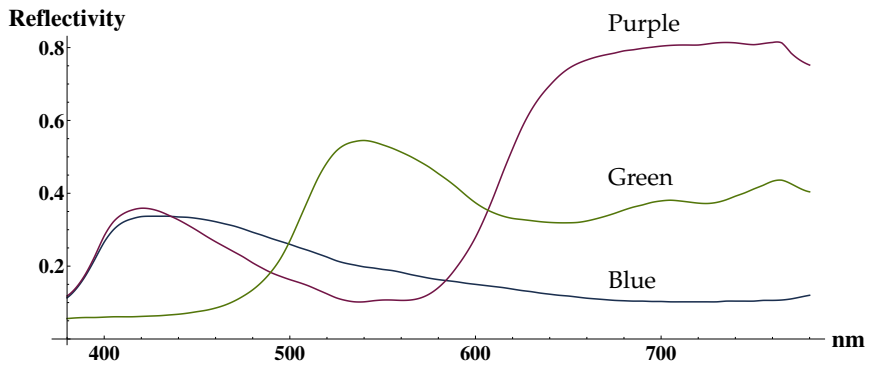

(a) Spectral reflectance $K_{d}(\lambda)$ for three color samples.

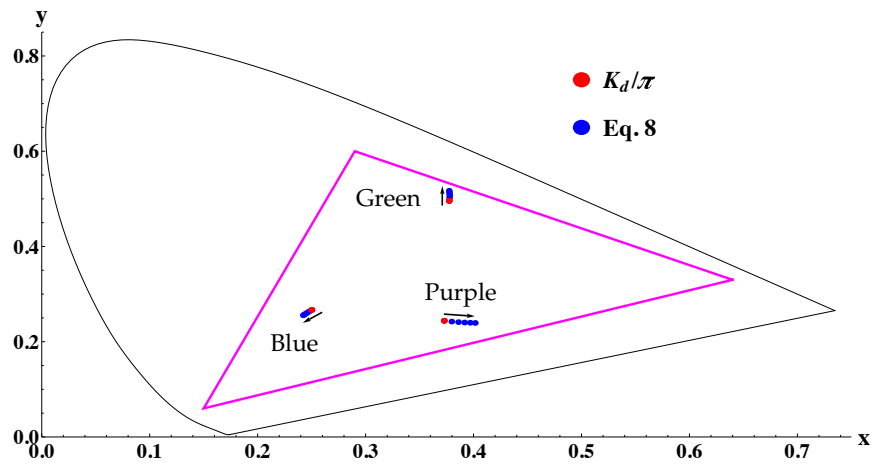

(b) Chromaticity variations for the three color samples given above and the color gamut PAL/SECAM, according to $n_{i}$ for values equal to $n_{i}=\{1.0,1.1,1.2,1.3,1.4,1.5\}$, with $\theta_{i}=65^{\circ}$. The arrows illustrate the variation direction when $n_{i}$ increases.

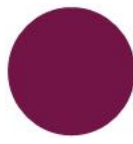

$n_{i}=1.0$

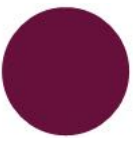

$n_{i}=1.1$

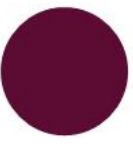

$n_{i}=1.2$

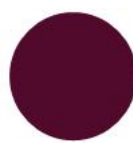

$n_{i}=1.3$

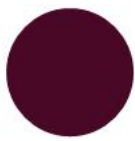

$n_{i}=1.4$

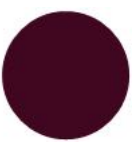

$n_{i}=1.5$ (c) Visual aspect of chomaticity variations according to $n_{i}$ for the purple color sample, with PAL/SECAM color gamut and $\theta_{i}=65^{\circ}$.

Fig. 5: $K_{d}(\lambda)$ and chromaticity variations according to $n_{i}$, for three samples from the Macbeth color checker.

tion 9) expresses an extension of the light beam from a medium of index $n_{1}$ to a less refringent medium of index $n_{0}<n_{1}$. The multiple reflections between the interface and the substrate lead to chromatic variations depending on $n_{i}$. Figure 5 a provides three spectral reflectances of the Macbeth color checker corresponding to CIE $x y$ values given in Figure 5b for normalized illuminant D65. This diagram shows that chromaticity actually varies according to $n_{i}$, providing darker and more saturated colors. Figure $5 \mathrm{c}$ illustrates colored disks with these variations.

Even with achromatic surfaces, raising values of $n_{i}$ increases specular reflections, and consequently decreases body reflections. Figure 6 shows the curves and a rendered 3D object with materials corresponding to a flat interfaced Lambertian surface, with varying values of $n_{i}$. Light inter-reflections between the interface and the substrate affect the object appearance depending on $K_{d} /\left(1-K_{d} r_{i}\right)$. In addition, the body reflected radiance also decreases when observation angles become grazing, contrary to Lambertian or rough Lambertian materials.
This interfaced Lambertian microfacet representation is by construction energy conserving. Note that this phenomenon can be observed on many measured materials (as shown in the supplemental material).

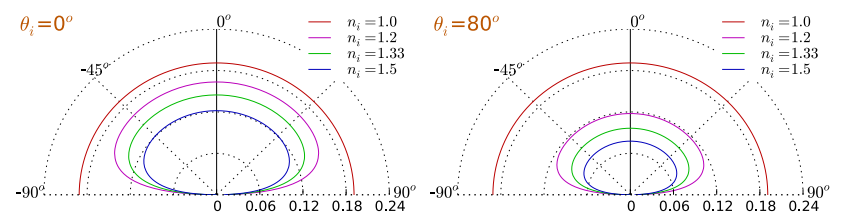

(a) Body reflection according to refractive index (the specular reflection peak is not illustrated here since it only corresponds to a dirac).

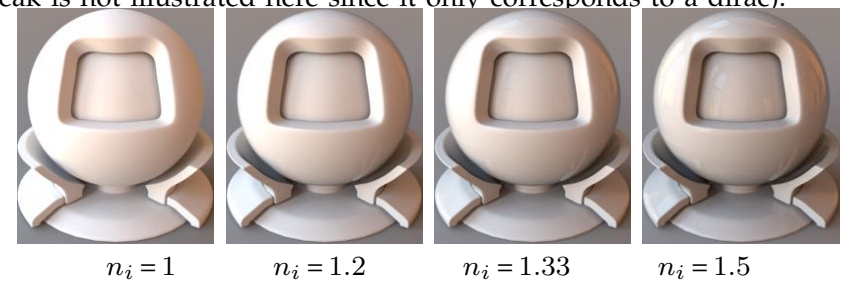

(b) Rendered images of a flat interfaced Lambertian BRDF, with various values of $n_{i}$

Fig. 6: (a) BRDF of a flat interfaced Lambertian surface with $K_{d}=0.6$ and $n_{i}=\{1.0,1.2,1.33,1.5\}$; (b) rendered images of the same BRDFs applied on a 3D object.

\subsection{Rough Interfaced Lambertian BRDF}

Using $f^{\mu}=f_{s}^{\mu}+f_{b}^{\mu}$ from Equation 5 in the integral of Equation 3 also provides a glossy and a matte component (resp. $f_{s}$ and $f_{b}$ ):

$$
f(\mathbf{i}, \mathbf{o}, \mathbf{n})=f_{s}(\mathbf{i}, \mathbf{o}, \mathbf{n})+f_{b}(\mathbf{i}, \mathbf{o}, \mathbf{n}) .
$$

The specular component corresponds to Equation 4 (coming from Equations 3 and 6), while the body reflection from Equations 3 and 9 becomes:

$$
\begin{aligned}
& f_{b}(\mathbf{i}, \mathbf{o}, \mathbf{n})=\frac{K_{d}}{\pi n_{i}^{2}\left(1-r_{i} K_{d}\right)} \times \\
& \int_{\Omega_{+}} T(\mathbf{i}, \mathbf{m}) T(\mathbf{o}, \mathbf{m}) D(\mathbf{m}) G(\mathbf{i}, \mathbf{o}, \mathbf{m}) \frac{|\mathbf{i m}|}{|\mathbf{i n}|} \frac{|\mathbf{o m}|}{|\mathbf{o n}|} d \omega_{m} .
\end{aligned}
$$

With this representation, several noticeable characteristics can be underlined:

- when $K_{d}=0$, this formulation corresponds to purely specular microfacets;

- when $n_{i}=1$, then $T=1$ and $r_{i}=0$, and it corresponds to purely Lambertian microfacets (Oren and Nayar's model [2]);

- when $\sigma=0$, the BRDF is a flat interfaced Lambertian surface;

- when $n_{i}=1$ and $\sigma=0$, the model is equivalent to a flat Lambertian material.

In this paper, we have employed various distributions and geometric attenuation factors from the literature.

Gaussian distribution of normals is essentially used in the Oren-Nayar model [2]. This distribution is employed 
in this paper only for comparison purposes, with the following isotropic formulation:

$$
D(\mathbf{m})=C e^{\left(-\theta_{m}^{2} / 2 \sigma^{2}\right)} /|\mathbf{m n}|,
$$

where $\sigma$ is the roughness parameter (expressed in radians) and $1 / C=2 \pi \int_{\theta_{m}=0}^{\pi / 2} e^{\left(-\theta_{m}^{2} / 2 \sigma^{2}\right)} \sin \theta_{m} d \theta_{m}$. Using such a distribution is not straightforward in practice due to the estimation of $C$. An analytical approximate version of $C$ that depends on $\sigma$ is proposed in the Appendix. Note that a correspondence with Beckmann or GGX distributions can be done with $\sigma_{\text {Beckmann } / G G X}=\sqrt{2} \sigma_{\text {Gauss }}$. We have employed this distribution for our approximate model in order to propose a solution compliant with Oren and Nayar's representation [2].

In this case, we also use the Torrance-Sparrow geometric attenuation factor $G(\mathbf{i}, \mathbf{o}, \mathbf{m})$ [15], which is mathematically well posed [27]:

$$
G(\mathbf{i}, \mathbf{o}, \mathbf{m})=\max \left(0, \min \left(1, \frac{2|\mathbf{i n}||\mathbf{m} \mathbf{n}|}{|\mathbf{i m}|}, \frac{2|\mathbf{o n}||\mathbf{m n}|}{|\mathbf{o m}|}\right)\right) .
$$

This factor assumes V-cavities much longer than large, which is somehow physically unrealistic and prone to discontinuities at grazing angles, as already noticed by several authors.

Beckmann distribution is often referenced in the literature [1], [10], [19]. It corresponds to a Gaussian distribution of microfacet slopes, and can be derived for anisotropic distributions [9], [13]:

$$
D(\mathbf{m})=\frac{e^{\left(-\tan ^{2} \theta_{m}\left(\frac{\cos ^{2} \varphi_{m}}{\sigma_{x}^{2}}+\frac{\sin ^{2} \varphi_{m}}{\sigma_{y}^{2}}\right)\right)}}{\pi \sigma_{x} \sigma_{y}|\mathbf{m n}|^{4}}
$$

where $\sigma_{x}$ and $\sigma_{y}$ correspond to the roughness parameters (standard deviations of slopes) associated with a local coordinate system. The well-known Cook-Torrance model [1] corresponds to the isotropic form of this distribution (for $\sigma_{x}=\sigma_{y}$ ), associated with the Torrance-Sparrow GAF [15]. However, several authors have shown that the representation proposed by Smith [32] is physicallyspeaking preferable [10], [11], [27]. It makes the assumptions that masking and shadowing are independent:

$$
G(\mathbf{i}, \mathbf{o}, \mathbf{m})=G_{1}(\mathbf{i}, \mathbf{m}) G_{1}(\mathbf{o}, \mathbf{m}) .
$$

Given that the GAF should be independent from the normal $\mathbf{m}$ of a microfacet, i.e., $G_{1}(\mathbf{u}, \mathbf{m})$ is not correlated with any other microfacet, even nearby, it leads to the following expression:

$$
G_{1}(\mathbf{u}, \mathbf{m})= \begin{cases}G_{1}(\mathbf{u}) & \text { when } \mathbf{u} \cdot \mathbf{m}>0 \\ 0 & \text { when } \mathbf{u} \cdot \mathbf{m} \leq 0\end{cases}
$$

with

$$
G_{1}(\mathbf{u})=\frac{\mathbf{u} \cdot \mathbf{n}}{\int_{\Omega_{u}^{+}} D(\mathbf{m})|\mathbf{u m}| d \omega_{m}} .
$$

Ashikhmin et al. [10] suggest a prior numerical integration for all directions $\mathbf{u}$. With the Beckmann distribution, this integral has an analytical expression [27] with
$G_{1}(\mathbf{u})=1 /(1+\Lambda(\mathbf{u}))$, where:

$$
\Lambda(\mathbf{u})=\frac{\operatorname{erf}(a)-1}{2}+\frac{1}{2 a \sqrt{\pi}} e^{-a^{2}},
$$

and $a=1 /\left(\alpha_{u} \tan \theta_{u}\right), \alpha_{u}=\sqrt{\sigma_{x}^{2} \cos ^{2} \varphi_{u}+\sigma_{y}^{2} \sin ^{2} \varphi_{u}}$. In the remainder of this paper, we will make the distinction between Gaussian and Beckmann distributions as described in the two previous paragraphs, although Beckmann also corresponds to a Gaussian.

GGX distribution [11], [33] has been extended to anisotropic distribution [27]:

$$
D(\mathbf{m})=\frac{1}{\pi \alpha_{x} \alpha_{y}|\mathbf{m n}|^{4}\left(1+\tan ^{2} \theta_{m}\left(\frac{\cos ^{2} \varphi_{m}}{\sigma_{y}^{2}}+\frac{\sin ^{2} \varphi_{m}}{\sigma_{y}^{2}}\right)\right)^{2}} .
$$

The associated GAF is also obtained using $G_{1}(\mathbf{u})=1 /(1+\Lambda(\mathbf{u}))$, with:

$$
\Lambda(\mathbf{u})=\frac{-1+\sqrt{1+1 / a^{2}}}{2}
$$

where $a=1 /\left(\alpha_{u} \tan \theta_{u}\right), \alpha_{u}=\sqrt{\sigma_{x}^{2} \cos ^{2} \varphi_{u}+\sigma_{y}^{2} \sin ^{2} \varphi_{u}}$.

\subsection{Multiple Reflections Between Microfacets}

Light multiple reflections between microfacet interface and body (Figure 4) are analytically handled by our model (Equation 9). However energy conservation also depends on light multiple reflections between microfacets. They should be explicitly accounted for. Figure 7 shows the variation of the integrated reflectance (integration over all outgoing and incident directions, given $K_{d}=1$ and $n_{i}=1.5$ ), according to $\sigma$. With $\sigma=0$, the surface corresponds to a flat interfaced Lambertian surface, which is energy conserving; the reflectance part due to light multiple reflections increases with roughness and cannot be neglected up to a certain value. For instance, when $\sigma=1.0$ it represents more than $20 \%$.

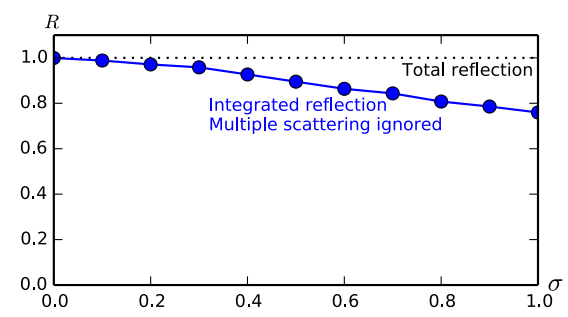

Fig. 7: Normalized total reflectance for a distribution of interfaced Lambertian microfacets according to $\sigma$, with a perfectly scattering background $\left(K_{d}=1\right.$, Beckmann distribution, and $n_{i}=1.5$ ). The blue curve illustrates the integrated body reflection without light multiple scattering between microfacets. The black dotted line illustrates the expected total reflection, with $R=1$.

Equation 3 stands for single-bounce reflections; multiple light reflections between microfacets have been neglected by many authors, and handled by Oren and 
Nayar [2], who consider the influence of two bounces of reflections with pure Lambertian microfacets, under the assumption of V-cavities (Figure 8).

Recently, Heitz et al. [34] discussed multiple light reflections with Smith representation and Monte Carlo integration. We show in this paper how it can be handled within V-cavities and interfaced Lambertian microfacets.

Given an incident light direction $\mathbf{i}$, the BRDF is the sum of all the contributions $f^{\infty}=f^{1}+f^{2}+\cdots$, where $f^{k}$ corresponds to the observed radiance (or reflectance with unitary irradiance) after $k$ reflections:

$$
\begin{aligned}
f^{\infty}(\mathbf{i}, \mathbf{o}, \mathbf{n}) & =\frac{d L^{\infty}(\mathbf{i}, \mathbf{o}, \mathbf{n})}{|\mathbf{i n}|} \\
& =\int_{\Omega+} \frac{R^{\infty}(\mathbf{i}, \mathbf{o}, \mathbf{m})}{|\mathbf{i n}|} \frac{|\mathbf{o m}|}{|\mathbf{o n}|} D(\mathbf{m}) d \omega_{m},
\end{aligned}
$$

where $d L^{\infty}$ is the total reflected radiance in direction o for a collimated light beam coming from direction $\mathbf{i}$. This formulation takes into account all light interactions with all possible microfacets complying with distribution $D$. $R^{\infty}(\mathbf{i}, \mathbf{o}, \mathbf{m})=\int_{S} L(\bar{x}) d \bar{x}$ is the total reflected radiance for all light paths $\bar{x}$ (from the path domain $S$ and $L(\bar{x})$ is the path contribution function) with at least one bounce inside a V-cavity represented by its normal $\mathbf{m}$.

$L(\bar{x})$ is estimated using path tracing; Equation 23 can be numerically integrated using Monte Carlo quadrature: First, a microfacet orientation is chosen according to importance sampling, depending on the probability density function (pdf) $D(\mathbf{m})|\mathbf{m n}|$; Second, a point $x_{i}$ is uniformly chosen on the microfacet; Third, a light path is built from a randomly chosen direction $i$. This path hits point $x_{i}$ from direction $\mathbf{i}$, reflects several times within the V-cavity, and finally gets out of the microsurface. This process can be directly integrated in any Monte Carlo path tracing system as explained in Section 4.1. With this method, shadowing and masking terms are automatically handled.

We propose to investigate the contributions of light multiple reflections within a virtual hemispherical sensor, subdivided such that every cell corresponds to the same solid angle (Figure 8).

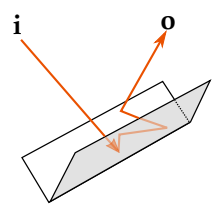

(a) Light path $\bar{x}$

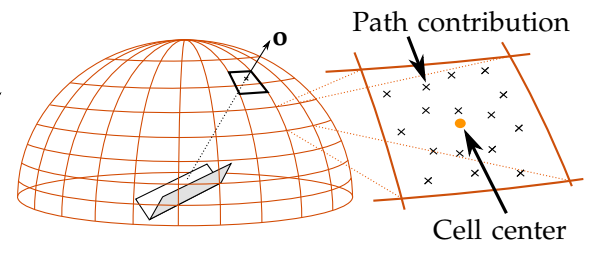

(b) Sensor and sensor cell
Fig. 8: Light inter-reflections within V-cavities: (a) Light path starts using a given direction $\mathbf{i}$ and interacts with the pair of microfacets before exiting in direction $\mathbf{o}$; (b) its contribution is collected on a hemispherical sensor subdivided in cells of equal solid angle; $f^{\infty}(\mathbf{i}, \mathbf{o}, \mathbf{n})$ is computed in each cell using density estimation based on all paths contributing to the cell.
Inside each sensor cell, density estimation is used to capture $f^{\infty}(\mathbf{i}, \mathbf{o}, \mathbf{n})$ where $\mathbf{o}$ is the center direction of the cell:

$$
f^{\infty}(\mathbf{i}, \mathbf{o}, \mathbf{n}) \approx \frac{\sum_{j=1}^{N} \frac{1}{|\mathbf{i n}|} L\left(\bar{x}_{j}\right) \frac{\left|\mathbf{o}_{\bar{x}_{j}} \mathbf{m}_{j}\right|}{\left|\mathbf{o}_{\bar{x}_{j}} \mathbf{n}\right|} D\left(\mathbf{m}_{j}\right)}{N_{\text {tot }} \Delta \omega_{o}},
$$

where $N_{t o t}$ is the total number of samples used to estimate Equation 23, $N$ is the number of paths contributing to the cell, $\mathbf{o}_{\bar{x}_{j}}$ is the outgoing direction of path $\bar{x}_{j}, \mathbf{m}_{j}$ is the sampled microfacet normal and $\Delta \omega_{o}$ the solid angle of any cell.

Figure 9 illustrates the effects of taking into account inter-reflections (two and multiple bounces). Simulations are done with one billion light paths and a sensor containing 3240 cells. Pure specular microfacets produce almost no additional reflected light; conversely, with body reflection and a rough surface, light inter-reflections have a significant impact on the BRDF.

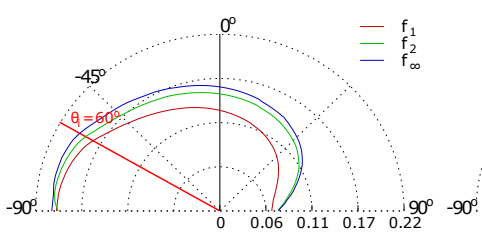

(a) $K_{d}=0.9, n_{i}=1.0, \sigma=0.6$

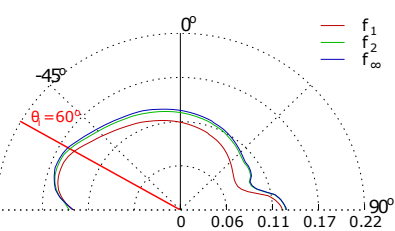

(b) $K_{d}=0.9, n_{i}=1.5, \sigma=0.6$
Fig. 9: V-cavities reflectance with inter-reflections, Beckmann distribution: $f_{1}$ corresponds to the BRDF with a single light bounce; $f_{2}$ corresponds to two light bounces; $f_{\infty}$ includes all bounces, using a Russian roulette strategy.

\section{BRDF INTEGRATION AND SAMPLING}

Interfaced Lambertian microfacet BRDFs are composed of two terms $f_{s}$ and $f_{b}$ (Section 3.2) that can be processed independently. Let us consider the rendering equation:

$$
L_{o}(x, \mathbf{o}, \mathbf{n})=L_{e}(x, \mathbf{o}, \mathbf{n})+\int_{\Omega_{+}} L_{i}(x, \mathbf{i}, \mathbf{n}) f(\mathbf{i}, \mathbf{o}, \mathbf{n})|\mathbf{i n}| d \omega_{i},
$$

where $x$ is the considered surface element location, $L_{o}(x, \mathbf{o}, \mathbf{n})$ corresponds to the outgoing radiance, $L_{e}(x, \mathbf{o}, \mathbf{n})$ is the self-emitted radiance, $L_{i}(x, \mathbf{o}, \mathbf{n})$ is the incident radiance coming from direction $\omega_{i}$, and $f(\mathbf{i}, \mathbf{o}, \mathbf{n})=f_{s}(\mathbf{i}, \mathbf{o}, \mathbf{n})+f_{b}(\mathbf{i}, \mathbf{o}, \mathbf{n})$. The glossy term corresponds to the well-known formulation provided by Equation 4, while the body part is directly derived from Equation 3, using the microfacet body contribution $f_{b}^{\mu}$ (Equation 13).

Unfortunately, this equation still requires numerical integration. The next section shows how it can be handled straightforwardly in any Monte Carlo rendering system, producing correct results without making use of any approximation. 


\subsection{Monte Carlo Integration}

Most renderers based on ray-tracing approaches make use of Monte Carlo integration for estimating the rendering equation. In those cases, BRDF importance sampling is used for reducing variance, as pointed out by many authors [11], [19], [35], [36].

Sampling the BRDF requires integrating Equation 3. The idea is to sample microfacet orientations using a stochastic process.

The specular component (Equation 4) corresponds to the usual Cook-Torrance formulation (with potentially different distributions and/or attenuation factors). It is thus managed with the existing importance sampling strategies [11].

The body component can also be estimated using stochastic sampling. Note that although microfacet reflectance and the resulting BRDF are smooth, uniform sampling is not efficient due to the distribution function $D$ that may introduce high frequencies (thin peaks and long tails), and consequently noise in the resulting images. Importance sampling should thus also be used, based on $D(\mathbf{m})|\mathbf{m n}|$.

Choosing between surface and body sampling is the first step. Ideally, $f_{s}$ and $f_{b}$ should be integrated to determine weighting. However, this process would be costly and inefficient in practice. Instead, we propose to simplify the problem considering a planar interface. Let us consider the total specular reflectance $R_{s}=r_{e}$ provided by Equation 11, and the total body reflectance $R_{b}=\frac{1}{n_{i}^{2}}\left(1-r_{e}\right)^{2} \frac{K_{d}}{1-K_{d} r_{i}}$, derived from the integration of Equation 9 over all incidence and observation directions (the mathematical details are provided in the Appendix); the proportion between $R_{s}$ or $R_{b}$ is used for choosing between the specular and the body direction: given a uniform random value $\xi_{0} \in\left[0,1\right.$, if $\xi_{0}<R_{s} /\left(R_{s}+R_{b}\right)$, a specular direction is sampled, otherwise a body direction is sampled.

Importance sampling the specular/glossy term has been discussed by many authors; Walter et al. [11] provide an overview on the subject. Based on the microfacet distribution, the pdf is given by $D(\mathbf{m})|\mathbf{m n}|$. The weight associated with the incoming direction is:

$$
w(\mathbf{i})=\frac{f_{s}(\mathbf{i}, \mathbf{o}, \mathbf{m}) D(\mathbf{m}) G(\mathbf{i}, \mathbf{o}, \mathbf{m})}{4|\mathbf{i n}||\mathbf{o n}| p_{o}(\mathbf{i})},
$$

with:

$$
p_{o}(\mathbf{i})=\operatorname{pdf}(\mathbf{m})\left\|\frac{\partial \omega_{h}}{\partial \omega_{o}}\right\|=\operatorname{pdf}(\mathbf{m}) \frac{1}{4|\mathbf{o m}|} .
$$

Body term sampling is more difficult since all microfacet contributions should be accounted for. We propose to transfer the BRDF integration in the rendering system directly. The Monte Carlo process can be applied to the whole rendering equation, including the analytical
BRDF:

$$
L_{o}(\mathbf{o}, \mathbf{n})=\int_{\Omega^{+}} L_{i}(\mathbf{i}, \mathbf{n}) \int_{\Omega^{+}} \hat{f}_{b}(\mathbf{i}, \mathbf{o}, \mathbf{m})|\mathbf{i n}| d \omega_{m} d \omega_{i},
$$

where $\hat{f}_{b}(\mathbf{i}, \mathbf{o}, \mathbf{m})=\frac{|\mathbf{i m}|}{|\mathbf{i n}|} f_{b}^{\mu}(\mathbf{i}, \mathbf{o}, \mathbf{m}) \frac{|\mathbf{o m}|}{|\mathbf{o n}|} D(\mathbf{m}) G(\mathbf{i}, \mathbf{o}, \mathbf{m})$, as stated in Equation 3.

This equation exhibits a four dimensional integral that can also be solved according to Monte Carlo importance sampling. Therefore, the process consists in selecting the incoming direction $\mathbf{i}$ according to a first importance sampling process, based on a pdf equal to $\mid$ in $\mid / \pi$, as well as a microfacet orientation $\mathbf{m}$ with a second importance sampling process based on a pdf equal to $D(\mathbf{m})|\mathbf{m n}|$. Anisotropy is straightforwardly handled by the chosen distribution of $D(\mathbf{m})$. The chosen orientation can also be used for the construction of a path within the corresponding V-cavity, for using $f_{2}$ or $f_{\infty}$, as described in Section 3.3.

Using the anisotropic distributions for Beckmann and GGX (Equations 16 and 21), sampling a microfacet $\mathbf{m}$ is given by the following equations. Let $\left(\xi_{1}, \xi_{2}\right)$ be two uniform random variables in $\left[0,1\left[{ }^{2}\right.\right.$; the value of $\varphi_{m}$ is identically sampled for all distributions:

$$
\varphi_{m}=\arctan \left(\frac{\sigma_{y}}{\sigma_{x}} \tan \left(2 \pi \xi_{1}\right)\right) \text {. }
$$

The value for $\theta_{m}$ depends on the chosen distribution, reversing the cumulative density function (cdf) associated with $\operatorname{pdf}(\mathbf{m})=D(\mathbf{m})|\mathbf{m n}|$.

With the Beckmann distribution:

$$
\theta_{m}=\arctan \left(\sqrt{\frac{-\log \left(\xi_{2}\right)}{\frac{\cos ^{2} \varphi_{m}}{\sigma_{x}^{2}}+\frac{\sin ^{2} \varphi_{m}}{\sigma_{y}^{2}}}}\right) .
$$

With the GGX distribution:

$$
\theta_{m}=\arctan \left(\sqrt{\frac{\xi_{1}}{\left(1-\xi_{1}\right)\left(\frac{\cos ^{2} \varphi_{m}}{\sigma_{x}^{2}}+\frac{\sin ^{2} \varphi_{m}}{\sigma_{y}^{2}}\right)}}\right) .
$$

Note that importance sampling a Gaussian distribution is not straightforward since the cdf cannot be analytically represented and thus inverted. A solution is to use another pdf with a similar shape, and we have chosen GGX (Equation 31), with $\sigma_{G G X}=\sqrt{2} \sigma_{\text {Gauss }}$.

\subsection{Body Approximate Model}

Some applications, including fitting parameters from measured data, or rasterization software, require a faster estimation of the BRDF. This is why we provide an approximate version of the body term, derived for several distributions, consistent with the one proposed by Oren and Nayar [2]. 
Let us note $f_{a}^{g}$ the approximate $L_{1}$ model defined by Oren and Nayar (with a Gaussian distribution). Our supplemental material provides a new approximate model $f_{a}^{b}$ for Lambertian microfacets, based on the Beckmann distribution, thus more consistent with many existing models [1], [9], [10].

Based on Equation 9, we propose the following approximation of the body reflection $f_{b}$ term:

$$
f_{b}(\mathbf{i}, \mathbf{o}, \mathbf{n}) \approx \frac{1}{n_{i}^{2}\left(1-K_{d} r_{i}\right)} \bar{T}(\mathbf{i}, \mathbf{n}) \bar{T}(\mathbf{o}, \mathbf{n}) F_{a},
$$

where $F_{a}$ describes the distribution, whose parameters $\left(\sigma_{x}, \sigma_{y}\right)$ define anisotropy:

$$
\begin{aligned}
\bar{T}(\mathbf{u}, \mathbf{n}) & =a T(\mathbf{u}, \mathbf{n})+b t_{01}, \\
t_{01} & =1-r_{e}, \\
F_{a} & =\left(1+\max \left(-1, c F_{a}^{\prime}\right)\right) f_{a}^{a *}(\mathbf{i}, \mathbf{o}, \mathbf{n}), \\
F_{a}^{\prime} & =-r_{e}(1-|\mathbf{i n}|)^{2}(1-|\mathbf{o n}|)^{2} \min \left(0,-H\left(\varphi_{i}, \varphi_{o}\right)\right), \\
H\left(\varphi_{i}, \varphi_{o}\right) & =d_{x y} \cos \left(\varphi_{o}\right) \cos \left(\varphi_{i}\right)+d_{y x} \sin \left(\varphi_{o}\right) \sin \left(\varphi_{i}\right),
\end{aligned}
$$

where $a, b, c, d_{x y / y x}$ are rational functions dependent on $\sigma_{x}, \sigma_{y}$, and $n_{i} ; f_{a}^{a *}(\mathbf{i}, \mathbf{o}, \mathbf{n})$ corresponds to the anisotropic Lambertian microfacet term: $f_{a}^{a g}(\mathbf{i}, \mathbf{o}, \mathbf{n})$ for a Gaussian distribution (anisotropic version of Oren and Nayar's approximation, that exactly corresponds to their isotropic representation when $\left.\sigma_{x}=\sigma_{y}\right)$; and $f_{a}^{a b}(\mathbf{i}, \mathbf{o}, \mathbf{n})$ for an anisotropic Beckmann distribution. The complete approximate model is given in the Appendix. We also propose a simpler version for isotropic distributions in the supplemental material.

This formulation is the exact formulation of a flat interfaced Lambertian surface when $\sigma_{x}=\sigma_{y}=0$ (Equation 9), and it equals the Oren-Nayar approximate model for a Gaussian distribution when $n_{i}=1$.

\section{Results ANd Discussion}

The interfaced Lambertian microfacets BRDF (referred to as $I L$ in the following) has been added as a plugin to the Mitsuba renderer [37], and the approximate version has been derived as a glsl shader, given in the supplemental material. Table 2 provides the configurations of distributions and attenuation factors used in this paper. All images have been produced with an environment lighting, except when specified otherwise in the caption.

TABLE 2: Configurations of distributions and attenuation factors used in this paper.

\begin{tabular}{|l|ll|}
\hline Notation & Distribution $(D)$ & Attenuation factor $(G)$ \\
\hline \hline Gauss, TS & Gaussian normals & Torrance-Sparrow \\
\hline Beckm, TS & Beckmann & Torrance-Sparrow \\
\hline Beckm, SB & Beckmann & Smith-Bourlier \\
\hline GGX & GGX & GGX GAF \\
\hline
\end{tabular}

Body reflection curves for a rough interfaced Lambertian material are shown in Figure 10, with several configurations for functions $D(\mathbf{m})$ and $G(\mathbf{m})$. The discontinuity corresponding to the Torrance-Sparrow GAF V-cavities essentially influences the BRDF at grazing incidence and/or observation angles. In addition, when $\sigma$ is low and/or when $n_{i}$ is close to 1 , the difference between Torrance-Sparrow and Smith GAFs tends to decrease. Note that the GGX distribution contains a higher tail compared to Beckmann or Gaussian distributions which tends to darken the material when $\sigma$ increases [11], [27]. This distribution also produces larger BRDF values at grazing observation angles for small values of $\sigma$ (Figure 10.c). Figure 11 shows the relative variations of distributions compared to Beckmann/SB, with the same parameters. With a Gaussian or a Beckmann distribution, the chosen GAF only slightly affects the appearance, and the difference is even smaller when $\sigma$ is small.

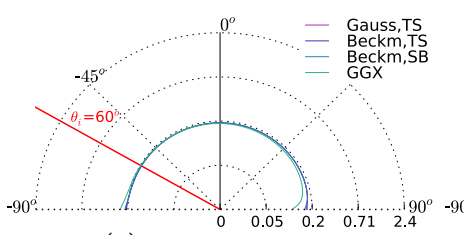

(a) $n_{i}=1.0, \sigma=0.1$

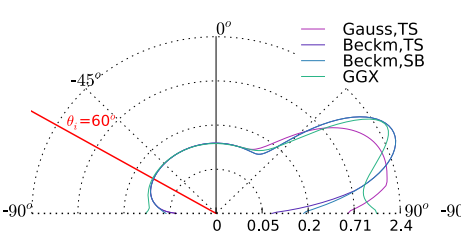

(c) $n_{i}=1.5, \sigma=0.1$

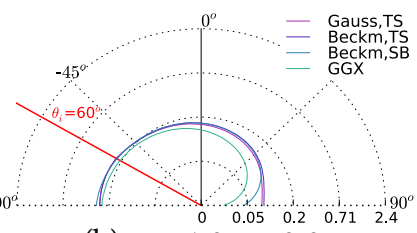

(b) $n_{i}=1.0, \sigma=0.6$

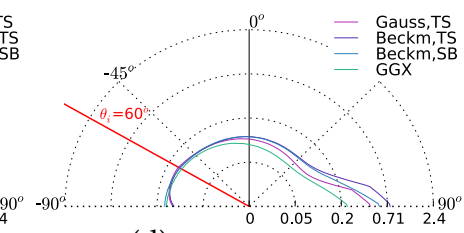

(d) $n_{i}=1.5, \sigma=0.6$
Fig. 10: Distributions and GAFs for various values of $n_{i}$ and $\sigma$, illustrated at $\theta_{i}=60^{\circ}(\log$ scale).

Monte Carlo rendering results are also provided, using path tracing. Figure 1 illustrates various materials, all modeled using interfaced Lambertian microfacets, either with the analytic version, or with the approximate one. Figures 12 and 13 illustrate rendered images with isotropic materials and various values of $n_{i}$ and $\sigma$. Anisotropic materials can be rendered with the same method, as shown in Figure 14. Note that even with $n_{i}=1.0$ (Lambertian microfacets), anisotropy remains visible. 


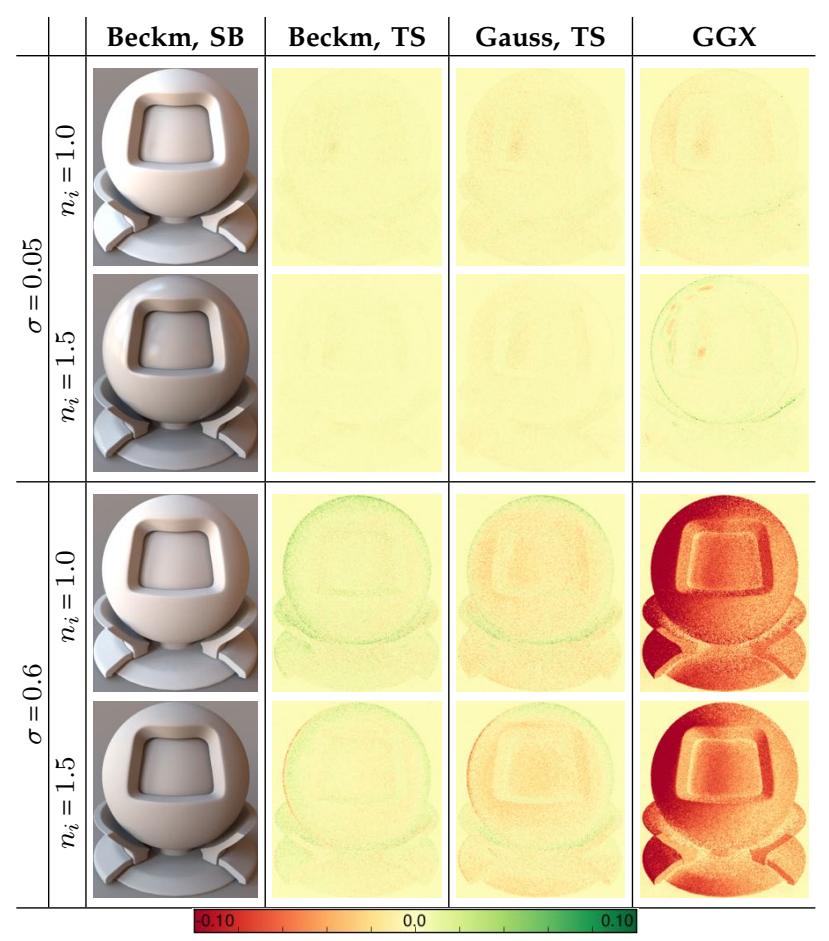

Fig. 11: Image differences for IL (without light multiple reflections between microfacets), with four distributions and GAFs, with $K_{d}=0.6$, two levels of roughness $\sigma$ and two values of $n_{i}$. The first column corresponds to the reference images. False colors correspond to the per-pixel difference value.

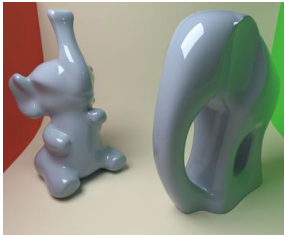

(a) $\sigma=0.001$

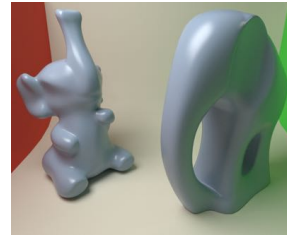

(b) $\sigma=0.1$

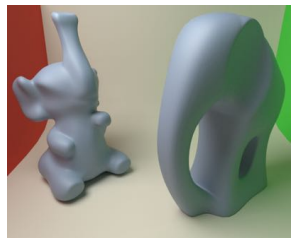

(c) $\sigma=0.3$
Fig. 12: Isotropic IL, Beckm, and SB elephants lit by two rectangular light sources, rendered using path tracing: $n_{i}=1.5$, for glossy materials and various roughnesses, clearly visible on the specular highlights.

Our approximate model corresponds to an alternative for interactive GPU applications or for fitting measured data, when the estimation of BRDF values needs to be performed faster. Figure 15 presents comparisons between our approximate model and Monte Carlo BRDF integration, with a Gaussian or a Beckmann distribution. The most important differences can be found for large values of $n_{i}$ and $\sigma$, and at grazing angles.

Visual comparisons are proposed firstly between IL and several configurations of opaque microfacet representations, and secondly between IL and multi-layered materials.

Figure 16 illustrates a comparison of our approach with two reflectance models already in Mitsuba, that handle both body and glossy reflections based on microfacets: Plastic (first column) uses a constant Lambertian term



Fig. 13: Appearance variations according to $n_{i}$ and $\sigma$, with a Beckmann distribution and Smith GAF. The bottom row corresponds to pure Lambertian microfacets, and the bottomleft image is an almost flat Lambertian surface.

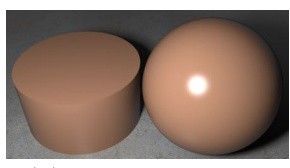

(a) $\sigma_{x}=0.1, \sigma_{y}=0.1$

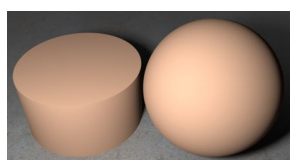

(d) $\sigma_{x}=0.1, \sigma_{y}=0.1$

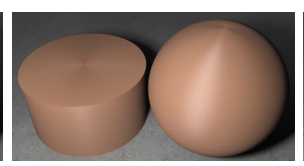

(b) $\sigma_{x}=0.2, \sigma_{y}=0.6$ Beckm, SB, $n_{i}=1.5$

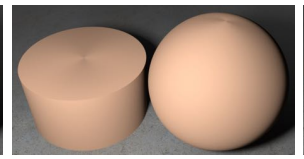

(e) $\sigma_{x}=0.1, \sigma_{y}=0.6$ Beckm, SB, $n_{i}=1.0$

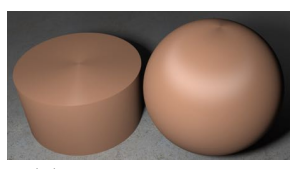

(c) $\sigma_{x}=0.6, \sigma_{y}=0.2$

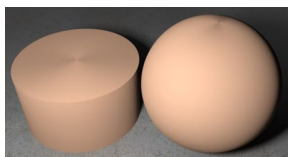

(f) $\sigma_{x}=0.6, \sigma_{y}=0.1$
Fig. 14: Anisotropic interfaced Lambertian microfacets, using a point light source, with (top) $n_{i}=1.5$ and (bottom) $n_{i}=1.0$.
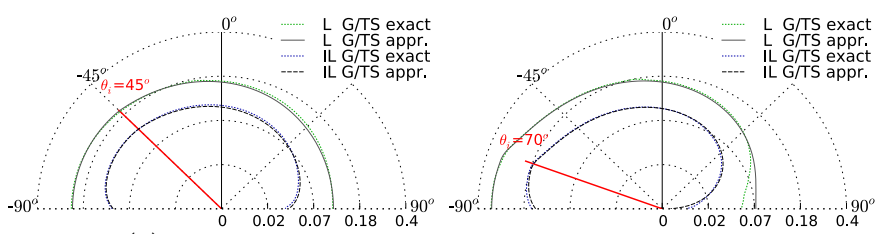

(a) Gaussian distribution, with $n_{i}=1.5$ and $\sigma=0.6$

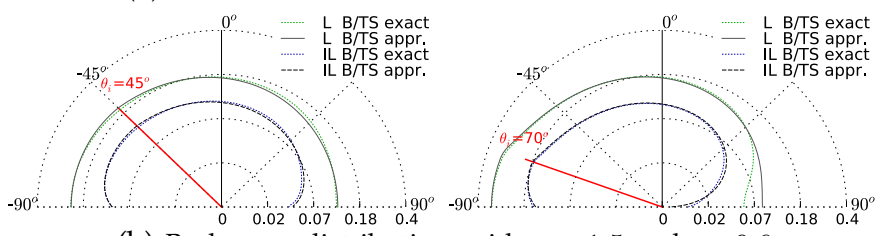

(b) Beckmann distribution, with $n_{i}=1.5$ and $\sigma=0.6$

Fig. 15: Comparison between Monte Carlo BRDF estimation of Lambertian (L) and interfaced Lambertian (IL) materials and our approximate model, with Gaussian (G) and Beckmann (B) distributions, and Torrance-Sparrow (TS) GAF (log scale). 
and Non Linear Plastic (denoted as Plastic2) implements the model proposed by Kelemen et al. [19] for energy conservation. The visual comparison points to several interesting observations: the Plastic material is much brighter than all the others due to the constant Lambertian term, as expected; $\mathrm{IL}_{2}$ is slightly brighter than $\mathrm{IL}_{1}$, which is visible when $\sigma$ increases, and the computation of $\mathrm{IL}_{\infty}$ brings only low additional energy, difficult to observe in practice in the images, though physically more accurate; neither Plastic nor Plastic2 models can reproduce the backscattering effects that appear when the substrate roughness increases, contrary to IL.

Figure 17 compares our model with the multi-layered representation proposed by Jakob et al. [12] (denoted as Layers) with a single layer for the rough interface (which is the best comparable configuration). Flat Lambertian surfaces (Lambert, Plastic2 and Layers) have darker silhouettes and a clearly visible geometry relief, while rough Lambertian materials (Rough Lambertian and IL) look flatter. Note that for this configuration, Plastic2 and Layers look very similar, and without backscattering. Multi-layered BRDFs proposed by Jakob et al. [12] estimate explicitly light multiple reflections between layers, but backscattering is again impossible to model since the authors consider the Lambertian substrate as flat, as discussed in Sections 1, 2.2 and in the supplemental material. We do not claim that our model should be considered as a substitute to multi-layer models, since they are able to handle many other BRDF configurations. However, it is a physically consistent representation for handling correctly opaque microfacet based dielectric materials.

It may also be tempting to combine Oren-Nayar (Gaussian distribution and Torrance-Sparrow GAF) for the body component and Cook-Torrance formulations using the same distribution and GAF, since backscattering would be handled. However such a combination is neither physically correct as explained in Section 2.2, nor equivalent to interfaced Lambertian microfacets, as shown in Figure 18.

Computational performance has also been compared with the models already existing in Mitsuba, as shown in Figure 16. Firstly, with path tracing, computation time is similar between the $I L_{1}$ exact model and the Mitsuba rough plastic models (corresponding to Plastic and Plastic2). When including multiple reflections between microfacets, the extra cost varies from 30 to $60 \%$, due to the additional ray paths. With path tracing, the exact BRDF estimation is as fast as the approximate one. This is due to the Monte Carlo process employed, that relies on only one microfacet during ray tracing, as explained in Section 4.1. For real-time applications, the approximate model should be used since the exact BRDF estimation would require sampling many microfacet orientations, which is much slower. In this case, the computation time is similar to Oren and Nayar's approximate representa-

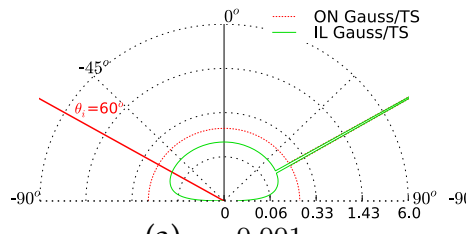

(a) $\sigma=0.001$

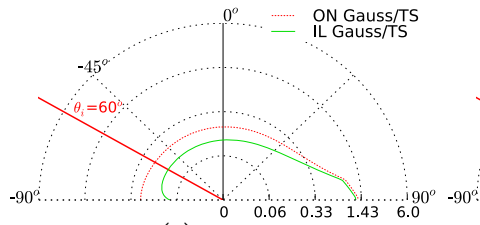

(c) $\sigma=0.3$

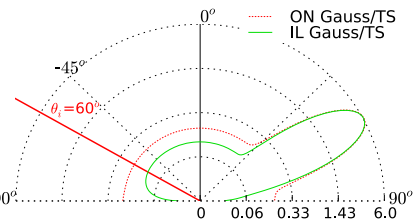

(b) $\sigma=0.05$

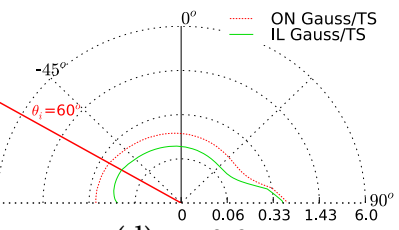

(d) $\sigma=0.6$
Fig. 18: Comparison between Oren-Nayar coupled with specular microfacets (Gaussian distribution and Torrance-Sparrow GAF) and interfaced Lambertian (IL, same distribution and GAF), with $K_{d}=0.6, n_{i}=1.5$, and various values of $\sigma$ (log scale).

tion.

Fitting measured data has been used to compare our model with actual BRDF data, where backscattering can be observed and body reflection actually decreases with larger observation angles, instead of being constant (see Figure 19). Such data cannot be precisely handled by the Cook-Torrance model because of its pure Lambertian behavior outside specular highlights, and backscattering cannot be represented by specular microfacets either. Figure 1 illustrates four rough interfaced Lambertian materials, including comparisons with measured materials and anisotropy. Several comparisons are illustrated in the supplemental material.

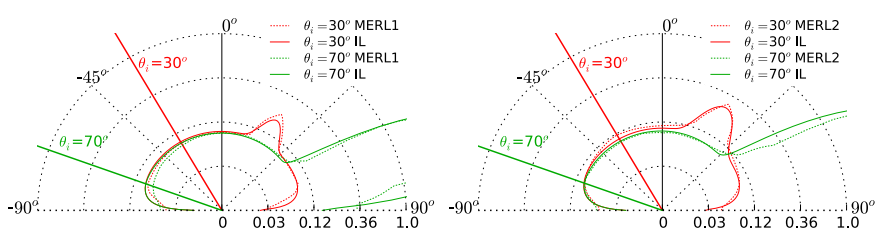

Fig. 19: Comparison between fitted (IL, Beckm, SB) and measured data from MERL database [38], for two incident directions $\theta_{i}=30^{\circ}$ and $\theta_{i}=70^{\circ}$ (log scale). MERL1 corresponds to specular-white-phenolic $\left(K_{d}=0.34, n_{i}=1.12, \sigma=0.065\right)$ and MERL2 corresponds to white-paint $\left(K_{d}=0.425, n_{i}=1.20\right.$, $\sigma=0.101)$. 


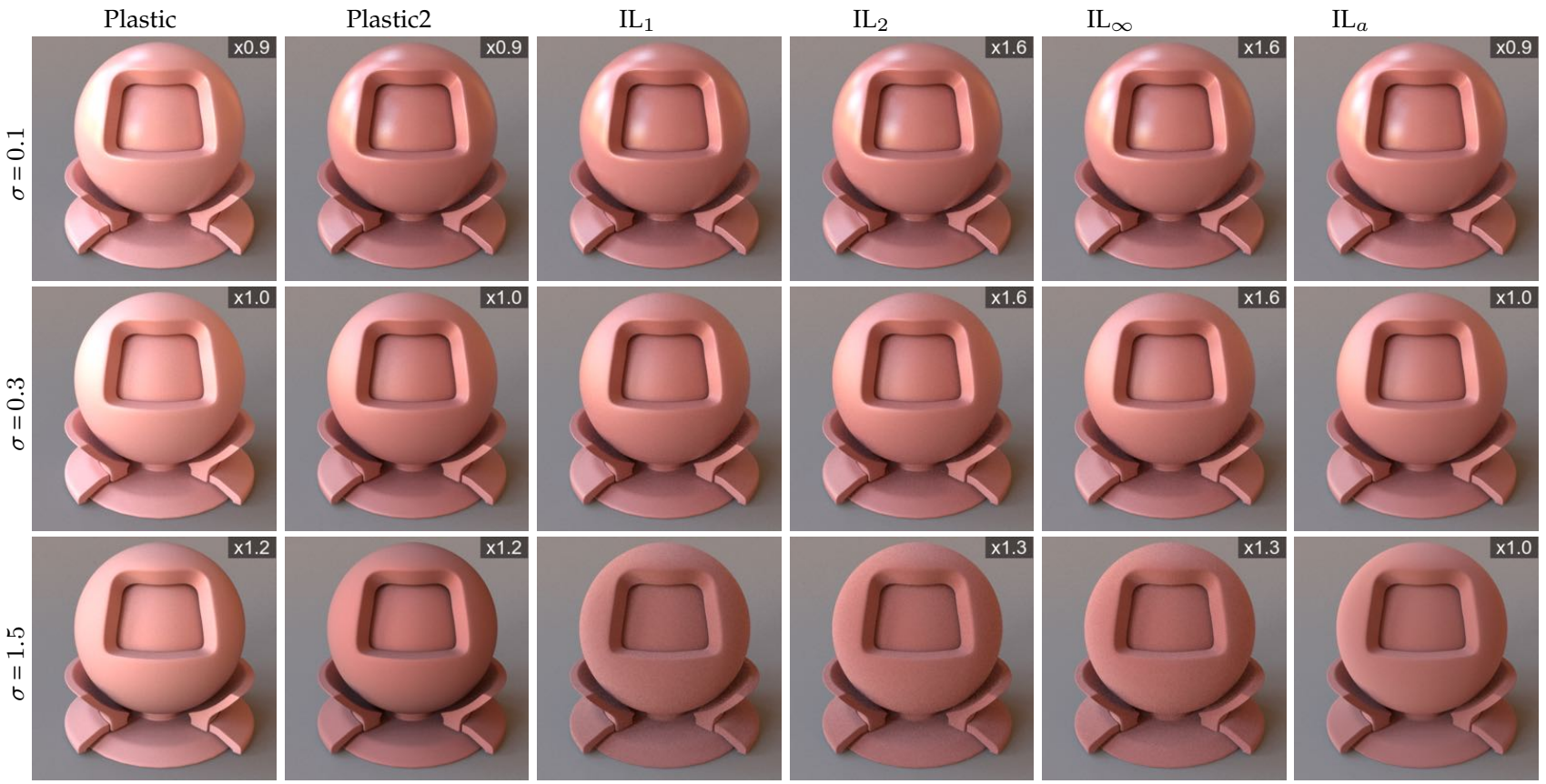

Fig. 16: Comparison between IL and other existing models, with $n_{i}=1.5$, and $\sigma=0.1$ for the first row, $\sigma=0.3$ for the second row, $\sigma=1.5$ for the third row. The first two models (columns) correspond to a Lambertian term for the body reflection, associated with Cook-Torrance. Plastic has a constant Lambertian value, while Plastic2 uses Kelemen et al. improvement [19]; $I L_{1}$ corresponds to IL model; $I L_{2}$ adds one reflection bounce between microfacets; $I L_{\infty}$ includes all reflections; $I L_{a}$ corresponds to our approximate model. All the model employ a Beckmann distribution associated with Smith GAF except for IL for which Torrance and Sparrow's GAF is used for estimating multiple reflections between microfacets. The reference computation time is given by IL, each other image contains the relative time in the top left corner.
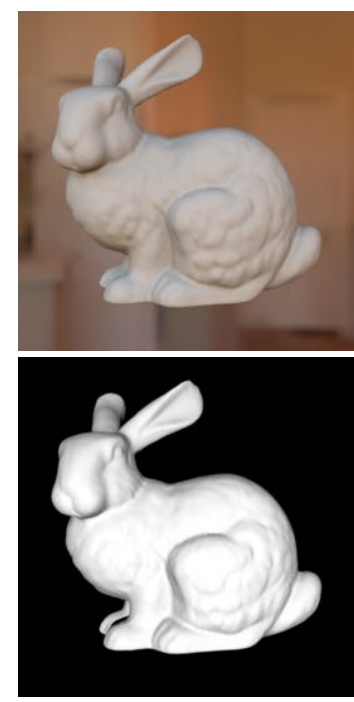

(a) Lambert
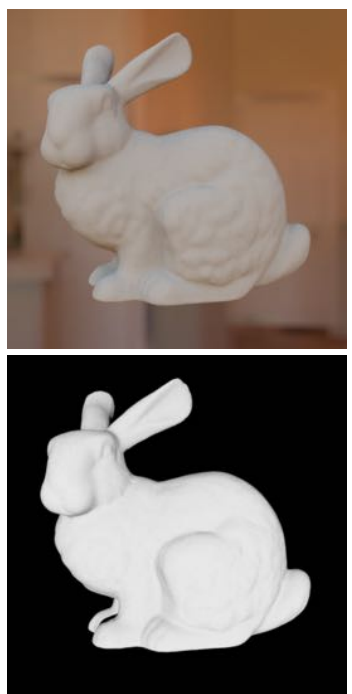

(b) Rough Lambertian
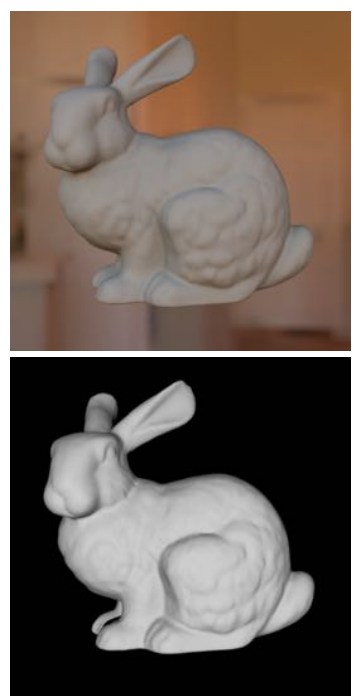

(c) Plastic2
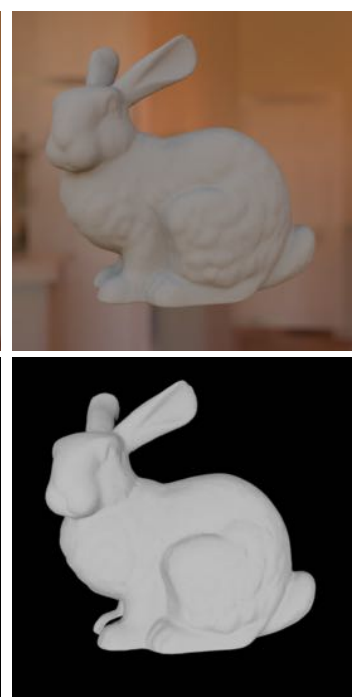

(d) $\mathrm{IL}_{\infty}$
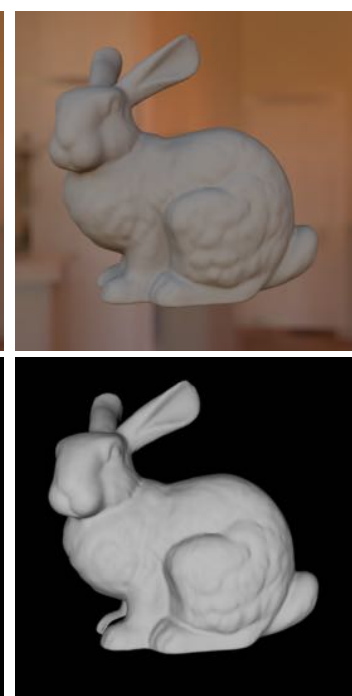

(e) Layers

Fig. 17: Comparisons between several models, including IL and multi-layered BRDFs: (a) flat Lambertian, (b) rough Lambertian [2], (c) Mitsuba non linear plastic [19], (d) IL with all reflections between microfacets using our approach with V-cavities, and (e) multi-layered representation from Jakob et al. [12]. For all models a Beckmann distribution is associated with Smith GAF except for IL for which Torrance and Sparrow's GAF is used for estimating multiple reflections between microfacets, $\sigma=0.9$ (except Lambert which is not concerned), and $n_{i}=1.5$ (except for Lambert and rough Lambertian which are not concerned). The top row uses an environment map illumination while the bottom row corresponds to a point light source placed at the observer position. Note that backscattering effect only appears with rough Lambertian and IL representations. 


\section{Conclusion}

This paper presents an analysis and implementation methods for interfaced Lambertian microfacet BRDFs, based on physical foundations. Our rendering methodology can be extended to any type of microfacet-based BRDFs for Monte Carlo based lighting simulation systems.

The presented model includes backscattering effects, anisotropy, and light multiple reflections, while generalizing Cook-Torrance [1], Oren-Nayar [2] and any intermediate combination. Furthermore, it relies on few parameters (refractive index, substrate reflectance and surface roughness) and can be straightforwardly integrated within any Monte Carlo based rendering system using importance sampling with any distribution and geometric attenuation factor. This model should not be considered as an alternative to multi-layered BRDF representations since it is designed specifically for handling interface refractive index changes in the case of opaque microfacets.

We also propose an approximate version (corresponding to two distributions) of this model that can be employed with interactive rendering systems, or fitting BRDF data, for avoiding numerical integration which remains time consuming.

Our method proposes an estimate of light interreflections between microfacets, using a V-cavity approximation is simple to implement. A method based on the Smith GAF has been proposed by Heitz et al. [34].

An interesting question concerns the correlation between the interface and the substrate roughnesses, since a single-roughness value constrains both specular peaks and body reflection, which is not always observed in measured materials. This is a harder challenge we wish to investigate.

Another interesting issue concerns the impact of all the BRDF parameters on the human visual system. Although physical and mathematical background is very important for lighting simulation, some models or sets of parameters seem very similar, and also highly depend on the lighting environment.

\section{REFERENCES}

[1] R. L. Cook and K. E. Torrance, "A reflectance model for computer graphics," ACM Transactions on Graphics, vol. 1, no. 1, pp. 7-24, january 1982.

[2] M. Oren and S. K. Nayar, "Generalization of Lambert's reflectance model," in ACM SIGGRAPH, july 1994, pp. 239-246.

[3] B. T. Phong, "Illumination for computer generated pictures," Communications of the ACM, vol. 18, no. 6, pp. 311-317, june 1975.

[4] J. F. Blinn, "Models of light reflection for computer synthesized pictures," in ACM SIGGRAPH, july 1977, pp. 192-198.

[5] R. R. Lewis, "Making shaders more physically plausible," Computer Graphics Forum, vol. 13, no. 2, pp. 109-120, may 1994.

[6] L. Claustres, M. Paulin, and Y. Boucher, "BRDF measurement modelling using wavelets for efficient path tracing," Computer Graphics Forum, vol. 22, no. 4, pp. 701-716, december 2003.

[7] S. Westin, J. Arvo, and K. E. Torrance, "Predicting reflectance functions from complex surfaces," in ACM SIGGRAPH, july 1992, pp. 255-264.

[8] E. P. F. Lafortune, S.-C. Foo, K. E. Torrance, and D. P. Greenberg, "Non-linear approximation of reflectance functions," in ACM SIGGRAPH, july 1997, pp. 117-126.

[9] G. J. Ward, "Measuring and modeling anisotropic reflection," in ACM SIGGRAPH, july 1992, pp. 265-272.

[10] M. Ashikhmin, S. Premoze, and P. Shirley, "A microfacet-based BRDF generator." in ACM SIGGRAPH, august 2000, pp. 65-74.

[11] B. Walter, S. R. Marschner, H. Li, and K. E. Torrance, “Microfacet models for refraction through rough surfaces," in Eurographics Symposium on Rendering, june 2007, pp. 195-206.

[12] W. Jakob, E. D'Eon, O. Jakob, and S. Marschner, "A comprehensive framework for rendering layered materials," in ACM SIGGRAPH, august 2014.

[13] M. Kurt, L. Szirmay-Kalos, and J. Křivánek, "An anisotropic brdf model for fitting and monte carlo rendering," in ACM SIGGRAPH, august 2010

[14] F.-K. Wu and C. wen Zheng, "Microfacet-based interference simulation for multilayer films," Graphical Models, vol. 78, pp. 26-35, march 2015.

[15] K. E. Torrance and E. M. Sparrow, "Theory for off-specular reflection from roughened surfaces," Journal of Opt. Soc. Am. vol. 57, no. 9, pp. 1105-1114, 1967.

[16] P. Callet, "Physically based rendering of metallic paints and coated pigments," in Visualization and Modelling, december 1995, pp. 287-301.

[17] — , "From fundamental optical properties of materials to visual appearance: metals, alloys, gilts, glasses, paints, photonics and biophotonics," in VIIIth Color Conference, september 2012, pp. 1625.

[18] P. Shirley, H. Hu, B. Smits, and E. Lafortune, "A practitioners" assessment of light reflection models," in PG '97: Proceedings of Pacific Graphics '97, october 1997.

[19] C. Kelemen and L. Szirmay-Kalos, "A microfacet based coupled specular-matte BRDF model with importance sampling," in Eurographics (short presentations), september 2001, p. 4

[20] B. van Ginneken, M. Stavridi, and J. J. Koenderink, "Diffuse and specular reflectance from rough surfaces," Applied Optics, vol. 37, no. 1, pp. 130-139, january 1998.

[21] A. Kienle and F. Foschum, "250 years lambert surface: does it really exist?" Opt. Express, vol. 19, no. 5, pp. 3881-3889, february 2011.

[22] A. Weidlich and A. Wilkie, "Arbitrarily layered micro-facet surfaces," in GRAPHITE '07, december 2007, pp. 171-178. 
[23] L. Simonot, R. D. Hersch, M. Hébert, and S. Mazauric, "Multilayer four-flux matrix model accounting for directional-diffuse light transfers," Applied Optics, vol. 55, no. 1, pp. 27-37, january 2016.

[24] L. Simonot, "A photometric model of diffuse surfaces described as a distribution of interfaced Lambertian facets," Applied Optics, vol. 48, no. 30, pp. 5793-5801, october 2009.

[25] M. Bagher, C. Soler, and N. Holzschuch, "Accurate fitting of measured reflectances using a Shifted Gamma micro-facet distribution," Computer Graphics Forum, vol. 31, no. 4, pp. 1509-1518, june 2012.

[26] J. Dupuy, E. Heitz, J.-C. Iehl, P. Poulin, F. Neyret, and V. Ostromoukhov, "Linear efficient antialiased displacement and reflectance mapping," in ACM SIGGRAPH ASIA, november 2013, pp. 211-222.

[27] E. Heitz, "Understanding the masking-shadowing function in microfacet-based brdfs," Journal of Computer Graphics Techniques (JCGT), vol. 3, no. 2, pp. 32-91, june 2014.

[28] F. E. Nicodemus, J. C. Richmond, J. J. Hsia, I. W. Ginsber, and T. Limperis, Geometrical consideration and nomenclature for reflectance. Final Report National Bureau of Standards, Washington, DC. Inst. for Basic Standards., 1977

[29] C. Bourlier, G. Berginc, and J. Saillard, "One and two-dimensional shadowing functions for any height and slope stationary uncorrelated surface in the monostatic and bistatic configurations," IEEE Transactions on Antennas and Propagation, vol. 50, no. 3, pp. 312324, march 2002.

[30] M. Elias, L. Simonot, and M. Menu, "Bidirectional reflectance of a diffuse background covered by a partly absorbing layer," Optics Communications, vol. 191, no. 1-2, pp. 1-7, 2001.

[31] R. Molenaar, J. J. ten Bosch, and J. R. Zijp, "Determination of kubelka-munk scattering and absorption coefficients by diffuse illumination," Applied Optics, vol. 38, no. 10, pp. 2068-2077, april 1999.

[32] B. Smith, "Geometrical shadowing of a random rough surface," IEEE Transactions on Antennas and Propagation, vol. 15, no. 5, pp. 668-671, september 1967.

[33] T. S. Trowbridge and K. P. Reitz, "Average irregularity representation of a rough surface for ray reflection," Journal of Opt. Soc. Am., vol. 65 , no. 5 , pp. 531-536, may 1975.

[34] E. Heitz, J. Hanika, E. d'Eon, and C. Dachsbacher, "Multiplescattering microfacet bsdfs with the smith model," INRIA, Tech. Rep., november 2015.

[35] E. Lafortune, "Mathematical models and monte carlo algorithms for physically based rendering," Ph.D. dissertation, Katholieke Universiteit Leuven, 1996.

[36] E. Veach, "Robust monte carlo methods for light transport simulation," Ph.D. dissertation, Stanford University, 1998.

[37] W. Jakob, "Mitsuba renderer," 2010, http://www.mitsubarenderer.org.

[38] W. Matusik, H. Pfister, M. Brand, and L. McMillan, "A data-driven reflectance model," ACM SIGGRAPH, vol. 22, no. 3, pp. 759-769, Jul. 2003.

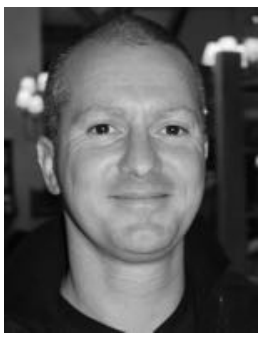

Daniel Meneveaux is Professor at the University of Poitiers (France) and the XLIM institute (CNRS UMR 7252). He was awarded a $\mathrm{PhD}$ degree in 1998 from the University of Rennes (France), and hired as Associate Professor in 1999 after a postdoctoral fellowship in the Imager laboratory at the University of British Columbia (Vancouver, Canada). His fields of interest are lighting simulation, image-based rendering, complex indoor scenes management, with extensions to topology-based geometric modeling and hierarchical representations.

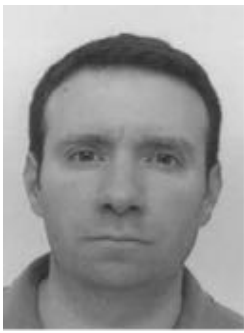

Benjamin Bringier is Associate Professor at the University of Poitiers (France) and the XLIM institute (CNRS UMR 7252). His research focuses on computer vision and image understanding. His interests concern acquisition and reproduction of color images for colorimetric and shape inspection with applications in roughness measurement, color management, and multi-spectral imaging. In 2002, he joined the French National Laboratory for Metrology and Testing (LNE) as $\mathrm{PhD}$ student and he received his PhD in 2005 from the University of Poitiers.

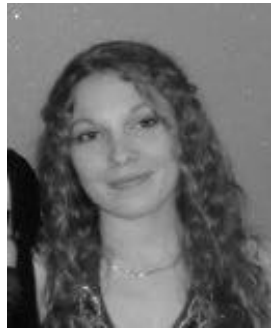

Emmanuelle Tauzia received her $\mathrm{PhD}$ degree at the University of Poitiers (France) and the XLIM institute (CNRS UMR 7252) in june 2016. Her research focuses on physically based bidirectional reflectance distribution functions, tridimensional reconstructions and BRDF acquisitions from images.

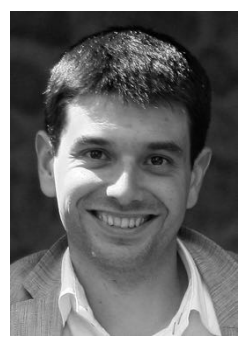

Mickaël Ribardière received is $\mathrm{PhD}$ degree in 2010 from the University of Rennes (France). He is currently Associate Professor at the University of Poitiers (France) and the XLIM institute (CNRS UMR 7252). His research interests are global illumination, lighting simulation for complex environments, and computer vision.



Lionel Simonot completed his $\mathrm{PhD}$ studies at the Centre de Recherche et de Restauration des Musées de France (C2RMF), located in the Palais du Louvre in Paris, where he studied light scattering by painting glazes. He obtained his PhD degree in 2002. Since 2003, he is Assistant Professor at the University of Poitiers (France) and the Pprime Institute (CNRS UPR 3346). His research activities focus on the optical properties of materials at different scales rough interfaces). (nanocomposite layers, scattering layers, flat or 